\title{
Influence of Small Satellites' Post-mission Disposal with Enlarging Effective Cross-sectional Area
}

\author{
By Yuya ARIYoshi ${ }^{1}$, Toshiya HANADA $^{2,3)}$ and Satomi KAWAmoto ${ }^{4)}$ \\ ${ }^{1)}$ National Astronomical Observatory of Japan, Mitaka, Japan \\ ${ }^{2)}$ Department of Aeronautics and Astronautics, Kyushu University, Fukuoka, Japan \\ ${ }^{3)}$ International Center for Space Weather Science and Education, Kyushu University, Fukuoka, Japan \\ ${ }^{4)}$ Japan Aerospace Exploration Agency, Chofu, Japan
}

(Received June 18th, 2013)

\begin{abstract}
Developers of small satellites may think of enlarging their effective cross-sectional area to minimize the long-term presence in the low Earth orbit region. However, the enlargement of their effective cross-sectional area causes the temporal increase of cumulative probability of accidental collisions. This paper evaluates the influence of small satellites' post-mission disposal by enlarging their cross-sectional area on the future population using an orbital debris environment evolutionary model. According to the result of the future projections, the small satellites' post-mission disposals by enlarging the effective cross-sectional area have the potential risk of increasing the accidental collision. However small satellites are inserted into orbit below $800 \mathrm{~km}$ in this paper, the fragments to be generated by collision expected to decay within short term. The effective number of objects below 2,000 km altitude is not different between the case with post-mission disposals and the case without post-mission disposals.
\end{abstract}

Key Words: Space Debris, Small Satellite, Post-mission Disposal

\section{Introduction}

The Inter-Agency Space Debris Coordination Committee's Space Debris Mitigation Guideline ${ }^{1)}$, the United Nations Committee on the Peaceful Uses of Outer Space's Space Debris Mitigation Guidelines ${ }^{2}$ and some national space agencies' internal standards or handbooks require spacecraft and launch vehicle orbital stages in the low-Earth orbit (LEO) region leave from their operational orbits and re-enter the Earth's atmosphere after the end of their mission. This guideline is so-called the 25-years rules because national space agencies' internal standards require orbital lifetime of spacecraft after their missions does not exceed 25 years. In general, conventional large satellites lower their altitude using their propulsion or reaction control system to comply with this guideline. On the other hand, small satellites, which are usually launched as secondary payloads, may not allow having any pressured vessel for orbit maneuverer, so that they cannot conduct such post-mission disposal (PMD) like the conventional large satellites do. Therefore, some small satellites developers manufacture devices to enlarging their effective cross-sectional area for shortening their orbital lifetime after their mission completion. ${ }^{3), 4)}$ However, the guideline also requests the limitation of probability of accidental collision. If the other conditions are same, larger objects have higher probability of accidental collision. Therefore, PMD with enlarging their cross-sectional area expects to raise their cumulative probability of collision during the PMD. Ariyoshi et al. ${ }^{5)}$ examined the cumulative probability of collision with or without PMD about typical small satellite and large satellite. Compared between small satellite with PMD and large satellite without PMD, the cumulative probability of small satellites with PMD is lower than that of large satellite without PMD. However, the influence of small satellite's PMD on future debris population is not clear, because the number of small satellites launch is increasing in recent year. This paper evaluates the influence of small satellites' PMD with enlarging their cross-sectional area on the future population using an orbital debris environment evolutionary model.

\section{Comparison of Cumulative Probability of Collision between Small and Large Satellites}

Before evaluating the influence of small satellites' PMD with enlarging their cross-sectional area on the future population, the results of cumulative probability of collisions during PMD examined in Ariyoshi et al. ${ }^{5)}$ is reviewed with some additional result. The results of large satellite' PMD with enlarging their cross-sectional area, small satellite' PMD with lower their perigee altitude and small/large satellite' PMD with lower their perigee and apogee altitude are added to the previous results. The typical small satellite before PMD is assumed as a $50 \mathrm{~cm}$ cubic satellite whose mass is 50 $\mathrm{kg}$. This assumed satellite has a $0.375 \mathrm{~m}^{2}$ average cross-sectional area, and the area-to-mass ratio is 0.0075 $\mathrm{m}^{2} / \mathrm{kg}$. The area-to-mass ratio of typical large satellite is assumed as $0.0085 \mathrm{~m}^{2} / \mathrm{kg}$ whose mass is $1750 \mathrm{~kg}$. This area-to-mass ratio is the average of launched satellites. The initial orbit of small and large satellite is assumed as a sun-synchronous orbit with an altitude of $700 \mathrm{~km}$. The conditions of PMD are summarized in Table 1. For 
evaluating the effectiveness of PMD, the case without PMD is also evaluated and named as Small 1 and Large 1. The case of PMD with enlarging their effective cross-sectional area is named as Small 2 and Large 2. The case of PMD with lower their perigee altitude is named as Small 3 and Large 3. The case of PMD with lower their perigee and apogee altitudes are named as Small 4 and Large 4.

Figure 1 demonstrates the cumulative probability of collision during descending their altitude with PMD. These results indicate that the cumulative probability of collision of Small 2 is lower than Large 1 at the time of re-entering the Earth's atmosphere. However, the small satellite with enlarging their effective cross-sectional area cause the temporal increase of cumulative probability of collisions. Therefore, the small satellites' PMD with enlarging their cross-sectional area have the potential risk of increasing the number of collision in future. In a similar fashion, the cumulative probability of Large 2 is higher than Large 1 during descending their altitude and the cumulative probability of collision at reentering the Earth's atmosphere is not different between Large 1 and 2 at the time of re-entering the Earth's atmosphere. Therefore, the enlarging the cross-sectional area is not appropriate for large satellite.

The PMD with lower their perigee/apogee altitude does not increasing the cumulative probability of collision temporally. The PMD with descending perigee and apogee altitude (Small 4 and Large 4) is higher than that with descending their perigee altitude. It is because that the spacecraft conducting PMD with lower the perigee and apogee altitude stay in the crowed region longer than that with lower the perigee altitude.

Table 1. Conditions of each post-mission disposal.

\begin{tabular}{lcccc}
\hline & & \multicolumn{3}{c}{ Conditions after PMD } \\
\cline { 3 - 5 } & PMD & $\begin{array}{c}\text { Effective } \\
\text { cross-sectional } \\
\text { area }\left[\mathrm{m}^{2}\right]\end{array}$ & $\begin{array}{c}\text { Perigee } \\
\text { height } \\
{[\mathrm{km}]}\end{array}$ & $\begin{array}{c}\text { Apogee } \\
\text { height } \\
{[\mathrm{km}]}\end{array}$ \\
\hline Small 1 & $\times$ & - & - & - \\
Small 2 & $\bigcirc$ & 1.3 & - & - \\
Small 3 & $\bigcirc$ & - & 538 & - \\
Small 4 & $\bigcirc$ & - & 615 & 615 \\
Large 1 & $\times$ & - & - & - \\
Large 2 & $\bigcirc$ & 46.375 & - & - \\
Large 3 & $\bigcirc$ & - & 555 & - \\
Large 4 & $\bigcirc$ & - & 623 & 623 \\
\hline
\end{tabular}

* - means that the condition is same as initial

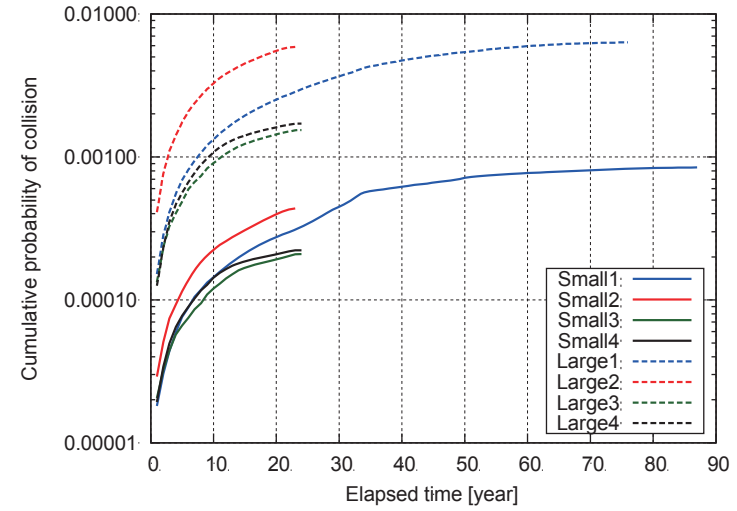

Fig. 1. Cumulative probability of collision during descending their altitude with various post-mission disposals.

\section{Future Projection}

\subsection{Orbital debris environment evolutionary model}

For evaluating the influence of small satellites' PMD on the future population, an orbital debris environment evolutionary model called NEODEEM (Near-Earth Orbital Debris Environment Evolutionary Model) is used. Japan Aerospace Exploration Agency and Kyushu University have jointly developed NEODEEM to evaluate future orbital debris population. This evolutionary model basically consists of orbit propagator, collision model and breakup model. The orbit propagator is based on Narumi and Hanada ${ }^{6}$ and considers the following perturbation forces: the geopotential, the gravity of the Sun and the Moon, the atmospheric drag and the solar radiation pressure. The probability of collision is calculated from each orbit. The NASA's standard breakup model $^{7), 8)}$ is used for modeling the fragments to be generated by accidental collisions.

\subsection{Future insertion assumption}

The launch of large spacecraft is conducted according to the traffic cycle of the years 2003 through 2010. The concept of small satellites' launch is different from large spacecraft, because the number of small satellites' launch is increasing in the recent years. According to the DePasquale and Charania $^{9)}$, it is expected that the number of small satellites inserted into orbits increase to 100 a year at maximum by 2020. Therefore, we have to assume the additional insertions of small satellites to evaluate the future population. There are three assumptions about the insertion of small satellites: how to insert small satellites into orbits and how many small satellites will be inserted with one rocket and how many rocket will be used for inserting small satellites for future projection.

Table 2. Number of rocket which launched small satellites from 2002-2010

\begin{tabular}{cccccc}
\hline & Total & $\begin{array}{c}\text { Secondary } \\
\text { payload }\end{array}$ & $\begin{array}{c}\text { From } \\
\text { shuttle }\end{array}$ & $\begin{array}{c}\text { Primary } \\
\text { payloads } \\
\text { (group) }\end{array}$ & $\begin{array}{c}\text { Primary } \\
\text { payload } \\
\text { (single) }\end{array}$ \\
\hline 2002 & 3 & 2 & 1 & 0 & 0 \\
2003 & 4 & 3 & 0 & 0 & 1 \\
2004 & 3 & 3 & 0 & 0 & 0 \\
2005 & 4 & 4 & 0 & 0 & 0 \\
2006 & 5 & 3 & 1 & 1 & 0 \\
2007 & 3 & 3 & 0 & 0 & 0 \\
2008 & 4 & 3 & 1 & 0 & 0 \\
2009 & 9 & 7 & 1 & 0 & 1 \\
2010 & 6 & 6 & 0 & 0 & 0 \\
\hline
\end{tabular}

Table 3. Number of small satellites $(<50 \mathrm{~kg})$ launched from 2002-2010

\begin{tabular}{cccccc}
\hline & Total & $\begin{array}{c}\text { Secondary } \\
\text { payload }\end{array}$ & $\begin{array}{c}\text { From } \\
\text { shuttle }\end{array}$ & $\begin{array}{c}\text { Primary } \\
\text { payloads } \\
\text { (group) }\end{array}$ & $\begin{array}{c}\text { Primary } \\
\text { payload } \\
\text { (single) }\end{array}$ \\
\hline 2002 & 9 & 8 & 1 & 0 & 0 \\
2003 & 9 & 8 & 0 & 0 & 1 \\
2004 & 9 & 9 & 0 & 0 & 0 \\
2005 & 5 & 5 & 0 & 0 & 0 \\
2006 & 10 & 3 & 4 & 3 & 0 \\
2007 & 14 & 14 & 0 & 0 & 0 \\
2008 & 10 & 9 & 1 & 0 & 0 \\
2009 & 25 & 21 & 3 & 0 & 1 \\
2010 & 23 & 23 & 0 & 0 & 0 \\
\hline
\end{tabular}


At first, the assumption about how to insert small satellites into orbit is explained. Table 2 categorizes the number of rocket for inserting small satellites into orbits with respect to the condition of insertion. This table indicates that most of the small satellites are launched as secondary payloads with a primary large satellite. Therefore, the small satellites to be inserted in the future are modeled like Fig. 2. It is assumed that small satellites are inserted into the same orbit of the primary spacecraft but the phase angle is different by 10 degrees between each satellite. The orbit of primary spacecraft is selected from the insertion history from 2003 to 2010 whose altitude is lower than $800 \mathrm{~km}$.

Table 3 summarizes the number of small satellites with respect to the condition of launch. According to the Tables 2 and 3, a rocket launched one small satellite as a secondary payload like Fig. 3. However, the number of small satellites with one rocket is also increasing in recent year. Therefore, based on the recent years' circumstance, the number of small

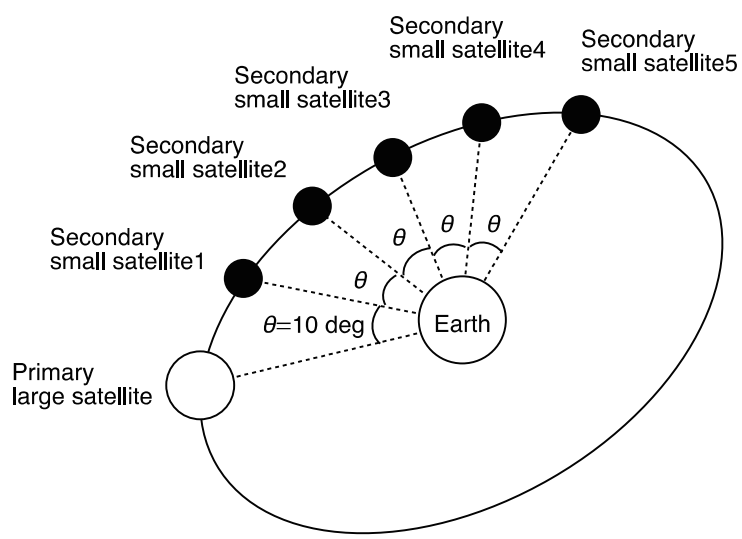

Fig. 2. The model of inserting small satellites as secondary payloads.

Table 4. Assumed number of rockets to be used for launching small satellites in NEODEEM.

\begin{tabular}{cc}
\hline Year & $\begin{array}{l}\text { Number of launches for small satellites } \\
\text { in NEODEEM }\end{array}$ \\
\hline 2010 & 5 \\
2011 & 2 \\
2012 & 10 \\
2013 & 11 \\
2014 & 13 \\
2015 & 14 \\
2016 & 16 \\
2017 & 16 \\
2018 & 18 \\
2019 & 19 \\
$2020-$ & 20 \\
\hline
\end{tabular}

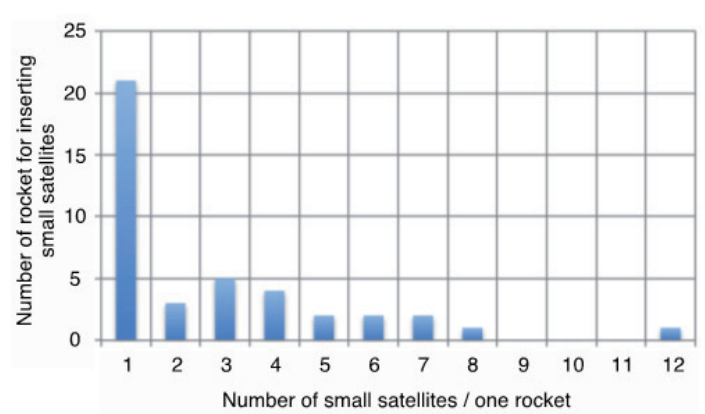

Fig. 3. Number of small satellites $(<50 \mathrm{~kg})$ with one rocket. satellites launched with one rocket is assumed as five.

The number of rockets for inserting small satellites is assumed. From 2010 to 2019, the number of small satellites' launch is assumed as Table 4, and after 2020 the number of rocket for launching small satellites is assumed as 100 a year. Each additional small satellite is assumed as $50 \mathrm{~cm}$ cubic whose mass is $50 \mathrm{~kg}$.

\subsection{Future projection condition}

Initial environment is as of May 1, 2009. This initial population includes larger than $10 \mathrm{~cm}$ objects whose perigee altitude is below $2,000 \mathrm{~km}$. The success rate of PMD is assumed as $90 \%$. Small satellites after their mission period conduct PMD with enlarging their cross-sectional area, whereas large satellites conduct PMD with descending their perigee altitude. Mission period is assumed as 8 years for large satellites and 2 years for small satellites. Future launch of spacecraft is assumed as sub-section 3.2. Furthermore, the case without additional small satellites launches and small satellite' PMD is also evaluated for comparison. The average of 60 Monte Carlo runs is the result of this simulation. 3.4. Result

The number of catastrophic collisions during next 200 years is shown in Fig. 4 and the number of non-catastrophic collisions is shown in Fig. 5. The collision location during 200 years is shown in Fig. 6. The number of catastrophic

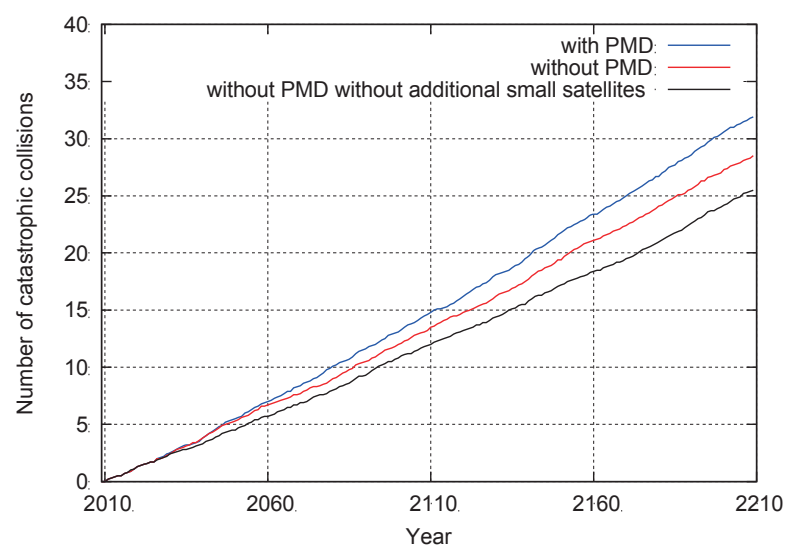

Fig. 4. Number of catastrophic collisions during 200 years.

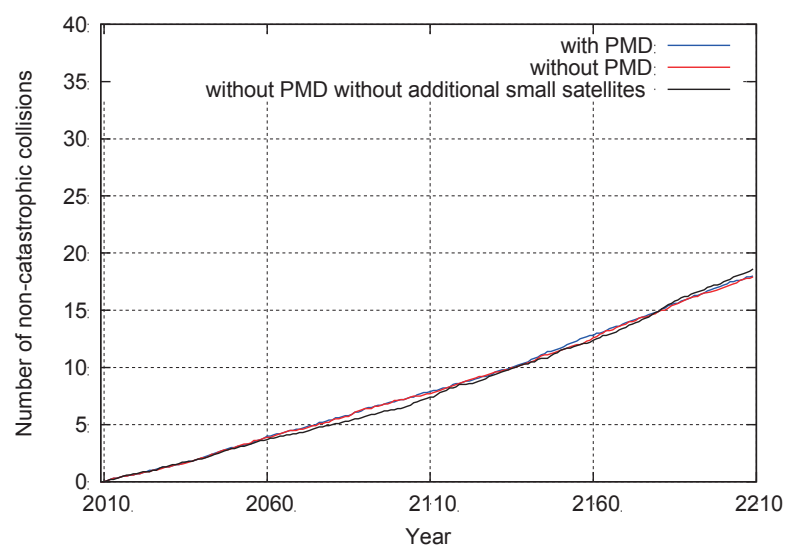

Fig. 5. Number of non-catastrophic collisions during 200 years. 


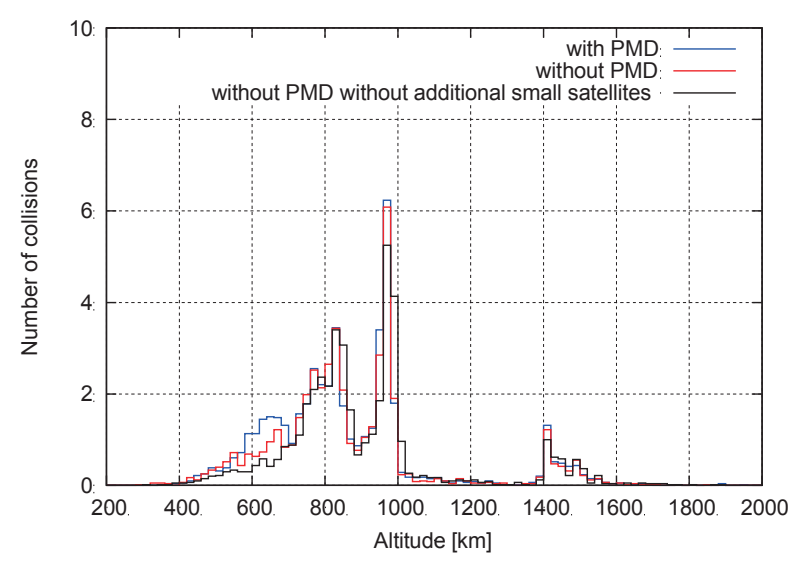

Fig. 6. Collision location between 200 and 2,000 km altitude during next 200 years.

collisions for the case with PMD is higher than that without PMD. This difference appears between 600-700 km altitudes. This is because PMD of small satellites conducts with enlarging their effective cross-sectional area, so that the probability of accidental collision temporally increases during the lower their altitude. The number of collision in the case with additional small satellites launches is higher than that without additional small satellites launches. It is not the influence of small satellites' PMD but that of additional small satellites' launches.

The effective number of objects in the LEO region $(200-2,000 \mathrm{~km})$ is shown in Fig. 7 . Fig. 8 presents the number of new fragments to be generated by collisions during 200 years. The population snapshot after 200 years is shown in Fig. 9. These are no difference between the results with PMD and without PMD. As shown in Fig. 7, the effective number of objects for the case with additional small satellites' launches is higher than that without additional small satellites' launches. However, as shown in Fig. 8, there are no differences among three cases with respect to the number of new fragments. Therefore, it is also because not the influence of small satellites' PMD but that of additional small satellites' launches. The population snapshot in Fig. 9 also indicates the same state.

According to these results, small satellite' PMD causes to raise the probability of collision and the number of catastrophic collision may increase at the altitude below the altitude of operating small satellites. However, small satellites are inserted into orbits below $800 \mathrm{~km}$ in this paper, and the fragments to be generated by collision expect to decay within short term.

\section{Conclusion}

This paper evaluated the influence of small satellites' post-mission disposal with enlarging the effective cross-sectional area on the future population. The results indicated that small satellites' PMD with enlarging their effective cross-sectional area bring about raising the probability of collision and the number of collision in the future. However, small satellites were inserted into orbits

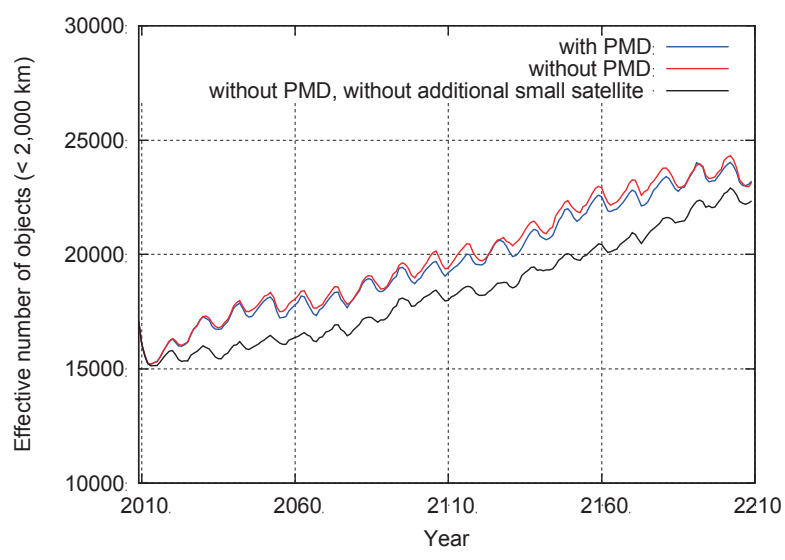

Fig. 7. Effective number of objects $(<2,000 \mathrm{~km})$ during 200 years.

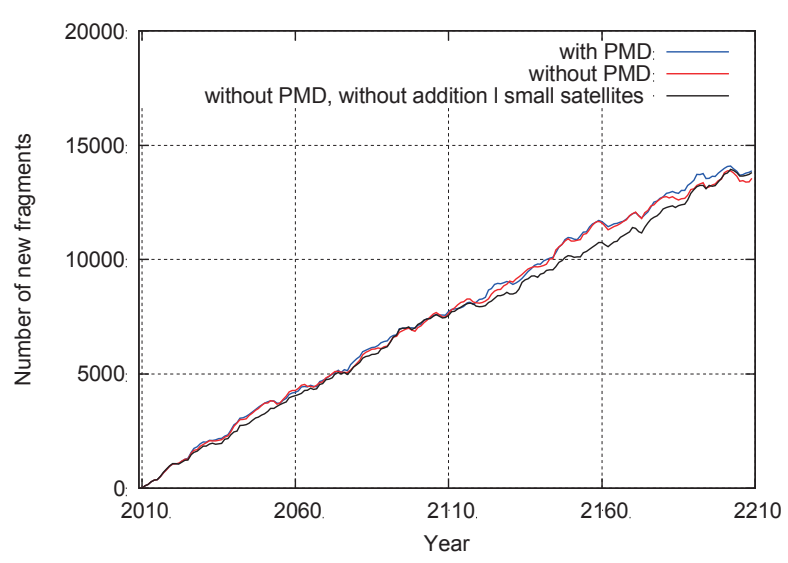

Fig. 8. Number of new fragments to be generated by collisions during 200 years.

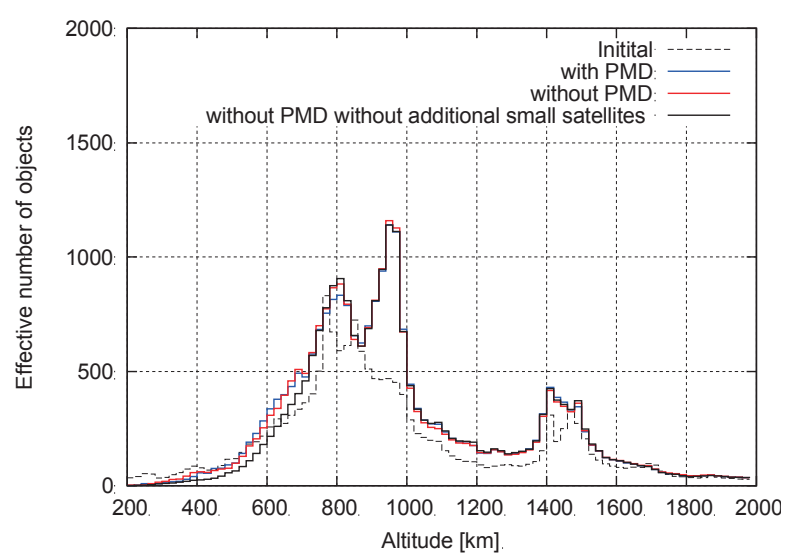

Fig. 9. Population snapshots between 200 and 2,000 km altitude at 2209.

below $800 \mathrm{~km}$ in this paper, so that the fragments to be generated by collision expected to decay within short term, and the effective number of objects below $2,000 \mathrm{~km}$ altitude is not different between the case with PMD and the case without PMD.

In these simulations, the area of a device for small satellites' PMD is included for calculating the probability of catastrophic 
collision. However, the device may be made of thin-film balloon or sail-like structure, and the collision between another spacecraft, and this PMD device may not lead to catastrophic breakup for impacted spacecraft. The separation of the main body and such a PMD device for calculating collision probability is the future work.

\section{References}

1) IADC Space Debris Mitigation Guidelines, IADC-02-01, September 2007.

2) Space Debris Mitigation Guidelines of the Scientific and Technical Subcommittee of the Committee on the Peaceful Uses of Outer Space, United Nations Publications, Sales No. E.99.I.17.

3) Onishi, S. et al.: Orbital Decay Accelerator: A Case of QSAT-EOS, The 3rd Nano-Satellite Symposium, NSS-03-0306, December 2011.
4) Kuwahara, T. et al., Development Status of Micro-satellite De-orbit Mechanisms for Active Prevention and Reduction of Space Debris, The 3rd Nano-Satellite Symposium, NSS-03-0304, December 2011.

5) Ariyoshi, Y. et al.: Are Small Satellites Hazardous in Comparison to Large Spacecraft?, Trans. JSASS Aerospace Tech. Japan, 11, pp. $1-5,(2013)$.

6) Narumi, T. and Hanada, T.: New Orbit Propagator to Be Used in Orbital Debris Evolutionary Models, Memories of the Faculty of Engineering Kyushu University, 67(4), pp.235-254, 2007.

7) Johnson, N.L. et al.: NASA's Breakup Model of EVOLVE 4.0, $A d v$. Space Res., 28(9), pp.1377-1384, 2001.

8) Anz-Meador, P.D., Matney M.J.: An Assessment of the NASA Explosion Fragmentation Model to $1 \mathrm{~mm}$ Characteristic Sizes, Adv. Space Res., 34, pp. 987-992,( 2004).

9) DePasquale, D. and Charania, A.: Nano/Microsatellite Launch Demand Assessment 2011, SpaceWorks Commercial, November 2011. 